\title{
An Automatic Clustering of Articles Using Dictionary Definitions
}

\author{
Fumiyo FUKUMOTO Yoshimi SUZUKI $\dagger$ \\ Dept. of Electrical Engineering and Computer Science, Yamanashi University \\ 4-3-11 Takeda, Kofu 400 Japan \\ \{fukumoto@skye, ysuzuki@suwa†\}.esi.yamanashi.ac.jp
}

\begin{abstract}
In this paper, we propose a statistical approach for clustering of articles using on-line dictionary definitions. One of the characteristics of our approach is that every sense of word in articles is automatically disambiguated using clictionary definitions. The other is that in order to cope with the problem of a phrasal lexicon, linking which links words with their semantically similar words in articles is introduced in our method. The results of experiments demonstrate the effectiveness of the proposed method.
\end{abstract}

\section{Introduction}

There has been quite a lot of research concerned with automatic clustering of articles or automatic identification of semantically similar articles(Walker, 1986), (Guthrie, 1994), (Yuasa, 1995). Most of these works deal with entirely different articles.

In general, the problem that the same word can be used differently in different subject domains is less problematic in entirely different articles, such as 'weather forecasts', 'medical reports', and 'computer manuals'. Because these articles are characterised by a larger number of different words than that of the same words. However, in texts from a restricted domain such as financial articles, e.g Wall Street Journal (WSJ in short) (Liberman, 1990), one encounters quite a large number of polysemous words. Therefore, polysemous words often hamper the precise classification of articles, each of which belongs to the restricted subject domain.

In this paper, we report an experimental study for clustering of articles by using on-line dictionary definitions and show how dictionarydefinition can use effectively to classify articles, each of which belongs to the restricted subject domain. We first describe a method for disambiguating word-senses in articles based on dictionary definitions. Then, we present a method for classifying articles ancl finally, we report some experiments in order to show the effect of the method.

\section{Related Work}

One of major approaches in automatic clustering of articles is based on statistical information of words in articles. Every article is characterised by a vector, each dimension of which is associated with a specific word in articles, and every coordinate of the article is represented by term weighting. Term weighting methods have been widely studicd in information retrieval rescarch (Salton, 1983), (Jones, 1972) and some of them are used in an automatic clustering of articles. Guthrie and Yuasa used word frequencies for weighting (Guthric, 1994), (Yuasa, 1995), and Tokunaga used weighted inverse document frequency which is a word frequency within the docnment divided by its frequency throughout the entire document collection (Tokunaga, 1994). The results of these methods when applied to articles' classification task, seem to show its effectiveness. However, these works do not seriously deal with the problem of polysemy.

The alternative approach is based on dictionary's information as a thesaurus. One of major problems using thesaurus categories as sense representation is a statistical sparseness for thesaurus words, since they are mostly rather uncommon words (Niwa, 1995). Yuasa reported the experimental results when using word frequencies for weighting within large documents were better results in clustering documents as those when EDR electronic dictionary as a thesaurus (Yuasa, 1995).

The technique developed by Walker also used dictionary's information and seems to cope with the discrimination of polysemy (Walker, 1986). He used the semantic codes of the Longman Dictionary of Contemporary English in order to determine the subject domain for a set of texts. For a given text, each word is checked against the dictionary to determine the semantic codes associated with it. By accumulating the frequencies for these senses and then ordering the list of categories in terms of frequency, the subject matter of 
the text can be identified. However, as he admits, a phrasal lexicon, such as Atlantic Seaboard, New England gives a negative influcuce for clustering, since it can not be regarded as units, i.c. each word which is the element of a phrasal lexicon is assigned to each semantic code.

The approach proposed in this paper focmses on these problems, i.e. polysemy and a plurasal lexicon. Like Guthrie and Yuasa's mothods, our approach adopts a vector representation, i.e. every article is characterised by a vector. However, while their approaches assign each coorelinate of a vector to each word in articles, we use a word (noun) of which sense is disambiguated. Our disambiguation method of word-senses is based on Niwa's method which used the similarity between two sentences, i.e. a sentence which contains a polysemous noun and a sentence of dictionarydefinition. In order to cope with Walker's problem, for the results of disambiguation technique, semantic relativeness of words are calculated, and semantically related words are grouped together.

We used WSJ corpus as test articles in the experiments in order to see how our method can effectively classify articles, each of which belongs to the restricted subject domain, i.c. WSJ.

\section{Framework}

\subsection{Word-Sense Disambiguation}

Every sense of words in articles which should be clustered is automatically disambiguated in advance. Word-sense disambiguation (WSD in short) is a scrious problen for NLP, and a varicty of approaches have been proposed for solving it (Brown, 1991), (Yarowsky, 1992).

Our disambignation method is based on Niwa's method which used the similarity between a sentence containing a polyscmons noun and a sentence of dictionary-definition. Let $x$ be a polysemous nown and a sentence $X$ be

$$
X: \quad \cdots, x_{-n}, \cdots, x_{-1}, x, x_{1}, \cdots, x_{n}, \cdots
$$

The vector representation of $X$ is

where $V\left(x_{i}\right)$ is

$$
V(X)=\sum_{i=-n}^{n} V\left(x_{i}\right)
$$

$$
V\left(x_{i}\right)=\left(M u\left(x_{i}, o_{1}\right), \cdots, M u\left(x_{i}, o_{m}\right)\right)
$$

Here, $M u(x, y)$ is the value of mutual information proposed by (Church, 1991). $o_{1}, \cdots, o_{n}$ (We call them basic words) are selected the 1000th most frequent words in the reference Collins English Dictionary (Liberman, 1990).

Let word $x$ have senses $s 1, s 2, \cdots, s p$ and the dictionary-definition of $s i$ be

$$
Y_{s i}: \quad \cdots, y_{-n}, \cdots, y_{-1}, y, y_{1}, \cdots, y_{n}, \cdots
$$

The similarity of $X$ and $Y_{s i}^{r}$ is measured by the inner product of their normalised vectors and is defined as follows:

$$
\operatorname{Sim}\left(X, Y_{s i}\right)=\frac{V(X) * V\left(Y_{s i}\right)}{\left|V(X) \| V\left(Y_{s i}\right)\right|}
$$

We infer that the sense of word $x$ in $X$ is si if $\operatorname{Sim}\left(X, Y_{s i}\right)$ is maximum among $Y_{s 1}, \cdots, Y_{s p}$.

Given an article, the procedure for WSD is applied to ach word (nomn) in an article, i.e. the sense of each noun is estimated using formula (1) ancl the word is replaced by its sense. Table 1 shows sample of the results of our disanbiguation method.

Table 1: The results of the WSD method

\begin{tabular}{l|l}
\hline Input & $\begin{array}{l}\text { A numbes of ma jor airlines adopted } \\
\text { continental airlines ... }\end{array}$ \\
$\begin{array}{l}\text { A number5 of major airlines } 1 \\
\text { adlopted continental2 airlines } 2 \ldots\end{array}$ \\
\hline
\end{tabular}

In 'Lable 1, underline signifies polysemons nonn. 'Output,' shows that ach noun is replaced by a symbol word which corresponds to cach sense of a word. Wo call 'Input' and 'Output' in 'Table 1, an original article and a new article, respectively.

Table 2: The definition of 'number'

\begin{tabular}{l|l}
\hline number1: & $\begin{array}{l}\text { Every number occupies a wique } \\
\text { position in a sequence. }\end{array}$ \\
number2: & He was not one of our number. \\
number3: & A telephone number. \\
number4: & She was number seven in the race. \\
number5: & A large number of people. \\
\hline
\end{tabular}

Table 2 shows the definition of 'number' in the Collins English Dictionary. 'number1' 'number5' are symbol words and show different senses of 'number'.

\subsection{Linking Nouns with their Semantically Similar Nouns}

Our method for classification of articles uses the results of disambiguation method. The problems here are:

1. The frecuency of every disambiguated noun in new articles is lower than that of every polysemous noun in original articles. For example, the frequency of 'number5' in Table 1 is lower than that of 'number'. Furthermore, some nouns in articles may be semantically similar with each other. For example, 'number'5' in Table 2 and 'sum4' in Table 3 are almost the same sense.

2. A phrasal lexicon which Walker suggested in his method gives a negative influence for classification.

\footnotetext{
${ }^{1}$ If all 'number' are used as 'number5' sense, the frecuency of 'number' is the same as 'number5'.
} 
Table 3: The definition of 's11m' in the dictionary

\begin{tabular}{|c|c|}
\hline suml: & The result of the addition of num- \\
\hline $\operatorname{sinm} 2$ & One or more columns or rows of \\
\hline sum3: & $\begin{array}{l}\text { numbers to be added. } \\
\text { The limit of the first } n \text { termis of a } \\
\text { converging infinite series as } n \text { tends } \\
\text { to infinity. }\end{array}$ \\
\hline sum4: & He borrows enormons s11ms. \\
\hline sum5: & The essence or gist of a matter. \\
\hline
\end{tabular}

In order to cope with these problems, we linked nouns in new articles with their semantically similar nouns. The procedures for linking are the following five stages.

\section{Stage One: Calculating $M u$}

The first stage for linking nouns with their semantically similar nouns is to calculate $M u$ between nown pair $x$ and $y$ in new articles. In order to get a reliable statistical clata, we merged every new article into one and used it to calculate $M u$. The results are used in the following stages.

Stage Two: Representing every noun as a vector

The goal of this stage is to represent every noun in a new article as a vector. Using a term weighting method, nouns in a new article would be represented by vector of the form

$$
v=\left(w_{1}, w_{2}, \cdots, w_{n}\right)
$$

where $w_{i}$ is the element of a new article and corresponds to the weight of the noun $w_{i}$. In our method, the weight of $w_{i}$ is the value of $M u$ between $v$ and $w_{i}$ which is calculated in Stage One.

\section{Stage Three: Measuring similarity between vectors}

Given a vector representation of nouns in new articles as in formula (2), a dissinilarity between two words (noun) $v_{1}, v_{2}$ in an article would be obtained by using formula (3). A dissimilarity measure is the degrec of deviation of the group in an $n$-dimensional Euclidean space, where $n$ is the number of nouns which co-occur with $v_{1}$ and $v_{2}$.

$$
\operatorname{Dis}\left(v_{1}, v_{2}\right)=\frac{\sum_{i=1}^{2} \sum_{j=1}^{n}\left(v_{i j}-\overline{g_{j}}\right)^{2}}{|\bar{g}|}
$$

$\bar{g}=\left(\overline{g_{1}}, \cdots, \bar{g}_{n}\right)$ is the centre of gravity and $|\bar{g}|$ is the length of it. A group with a smaller value of (3) is considered semantically less deviant.

\section{Stage Four: Clustering method}

For a set of nouns $w_{1}, w_{2}, \cdots, w_{n}$ of a new article, we calculate the semantic deviation value of all possible pairs of nouns.

Table 4 shows sample of the results of nouns with their semantic deviation values.
Table 4: Pairs of nouns with Dis $\left(v_{1}, v_{2}\right)$ values

\begin{tabular}{lll}
\hline \multicolumn{3}{c}{ BBK } \\
\hline 0.125 & share1 & company1 \\
0.140 & giorgio & di \\
0.215 & shares2 & share2 \\
0.262 & share2 & company 1 \\
$\ldots$ & $\ldots$ & $\ldots$ \\
0.345 & new 3 & york1 \\
$\ldots$ & $\ldots$ & $\ldots$ \\
\hline
\end{tabular}

In Table 4, 'BBK' shows the topic of the article which is tagging in the WSJ, i.e. 'Buybacks'. The value of Table 4 shows the semantic deviation value of two nouns ${ }^{2}$.

The clustering algorithm is applied to the sets shown in Table 4 and produced a set of semantic clusters, which are ordered in the ascending order of their semantic deviation values. We adopted non-overlapping, group average method in our clustering technique (Jardine, 1991). The sample results of clustering is shown in Table 5.

'Table 5: Clustering results of 'BBK'

\begin{tabular}{ll}
\hline 0.125 & [share1 company1] \\
0.140 & [giorgio di] \\
0.215 & [shares 2 share2] \\
0.251 & [share1 company1 share2 shares2] \\
$\cdots$ & $\cdots$
\end{tabular}

The value of Table 5 shows the semantic deviation value of the cluster.

Stage Five: Linking nouns with their semantically similar nouns

We selected different 49 articles from 1988, 1989 WSJ, and applied to Stage One Four. From these results, we manually selected clusters which are judged to be semantically similar. For the selected clusters, if there is a nomn which belongs to several clusters, these clusters are grouped together. As a result, each cluster is added to a sequential number. The sample of the results are shown in Table 6.

Table 6: The results of Stage Five

\begin{tabular}{l|l}
\hline Seq. num & Semantically similar nouns \\
\hline word $d_{1}:$ & bank3, banks3 \\
won $d_{2}:$ & canada3, canada4 \\
word $d_{3}:$ & American1, express 1 \\
word $d_{4}:$ & co., corp., company $1 \ldots$ \\
wor $d_{5}:$ & August, June, July, Sept. Oct. $\cdots$ \\
$\ldots$ & new2 york2 \\
$\cdots$ & $\ldots$ \\
\hline
\end{tabular}

\footnotetext{
${ }^{2}$ In Table 4, there are some nouns which are not added to the number, ' 1 ' $\sim$ '5', e.g. 'giorgio', 'di'. This shows that for these words, there is only one meaning in the dictionary.
} 
'Seq. num' in Table 6 shows a secquential number, 'word $d_{1}, \ldots$, ,word ${ }_{m}$ ' which are added to the group of semantically similar nonns'. Table 6 shows, for example, 'new2' and 'york2' are semantically similar and form a phrasal lexicon.

\subsection{Clustering of Articles}

According to Table 6 , frequency of every word in new articles is counted, i.e. if a work in a new article belongs to the group shown in Table 6 , the word is replaced by its representative number 'word $i$ 'and the frequency of 'word $d_{i}$ is connted. For example, 'bank3' and 'banks3' in a new article are replaced by 'word $i$ ', and the frecunency of "word ${ }_{i}$ "equals to the total number of frecueney of 'bank3' and 'banks3'.

Using a term weighting method, articles would be represented by vectors of the form

$$
A=\left(w_{1}, w_{2}, \cdots, w_{n}\right)
$$

where $w_{i}$ corresponds to the weight of the nonn i. The weight is nsed to the frecuency of noun. Given the vector representations of articles as in formula (4), a similarity between $A_{i}$ and $A_{j}$ are calculated using formula (1). The greater the value of $\operatorname{Sim}\left(A_{i}, A_{j}\right)$ is, the nore similar these two articles are. The clustering algorithen which is described in Stage Four is applied to carch pair of articles, and produces a set of clusters which are ordered in the descending order of their semantic: similarity values.

\section{Experiments}

We have conducted four experiments, i.c. 'Lreq", 'Dis', 'Jink', and 'Method' in oreler to examine how WSD method and linking words with their semantically similar words(linking nothod in short) affect the clustering results. 'Treel' is frequencybased experiment, i.e. we nse word frequency for weighting and do not use WSD and linking methods. 'Dis' is concerned with disambiguation-based experiment, i.e. the clustering algorithm is applied to new articles. 'Tink' is concerned with linking-based experiment, i.e. wo applied linking method to original articles. 'Method' shows our proposed method.

\subsection{Data}

The training corpus we have used is the 1988, 1989 WSJ in $\Lambda$ CL/DCI CD-ROM which consistis of about 280,000 part-of-spech tagged sentences (Brill, 1992). From this corpus, we selected at random 49 different articles for test data, each of which consists of 3,500 sentences and has different topic name which is tagging in the WSI. We classified 49 articles into eight categories, eg.

\footnotetext{
${ }^{3}$ In our experiments, $m$ equals to 238 .
}

'market news', 'food- restaurant', cte. 'The dictiothary we have used is Collins English Dictionary in $\hat{A C I} / \mathrm{DCI} C D-\mathrm{ROM}$.

In WSD method, the co-occurrence of $x$ and $y$ for calculating $M u$ is that the two words $(x, y)$ appear in the training corpus in this order in a window of 100 words, i.c. $x$ is followed by $y$ within a 100-word distance. This is because, the larger window sizes might be considered to be useful for extracting semantic relationships between nonns. Busir: words are selected the 1000th most frequent words in the reference Collins English Dictionary. 'I'he length of a sentence $X$ which contains a polysemons nomn and the length of a sentence of dictionary-definition are maximum 20 words. For ach polysemous nom, we solected the first top 5 definitions in the dictionary.

In linking methorl, a window size of the cooccurrence of $x$ and $y$ for calculating $M u$ is the sannc as that in WSD method, i.e. a window of 100 words. Wo selected $969 \sim 9128$ different (nom, 11011n) pairs for ach article, $377 \sim 1259$ differ rnt nowns on condition that frepuencies and $M u$ are not low $(f(x, y) \geq 5, M u(x, y) \geq 3)$ to pernit a reliable statistical analysis ${ }^{4}$. As a result of Stage Four, we manually sclected clusters which are juclged to be semantically similar. As a result, we selected clusters on condition that the threshold value for similarity was 0.475 . For the selected ansters, if there is a noun which belongs to sevcral clusters, these elusters are grouped togetlees. As a result, wo obtained 238 clusters in all.

\subsection{Results of the experiments}

The results are shown in Table 7.

Table 7: The results of the experiments

\begin{tabular}{cc|cccc}
\hline Artiche & Nurn & Fieq & Link & Dis & Method \\
\hline 5 & 10 & 4 & 4 & 5 & 8 \\
10 & 10 & 4 & 6 & 6 & 9 \\
15 & 10 & 7 & 7 & 7 & 8 \\
20 & 10 & 6 & 6 & 6 & 6 \\
\hline Total & 10 & 21 & 23 & 24 & 31 \\
$(\%)$ & $(-)$ & $(52.5)$ & $(57.5)$ & $(60.0)$ & $(77.5)$ \\
\hline
\end{tabular}

In Table 7, 'Article' means the number of articles which are selected from test data. 'Num' means the number for each 'Article', i.e. we sclected 10 sets for each 'Article'. 'Freq', 'Link', 'Dis', and 'Method' show the number of sets which are clustered correctly in each experiment.

The sample results of 'Article $=20$ ' for cach experiment is shown in Figure $1,2,3$, and 4.

In Figure 1, 2, 3, and 4, the $X$-axis is the similatity value. Abbreviation words in cach Figure and categories are shown in Table 8.

\footnotetext{
${ }^{4}$ Here, $f(x, y)$ is the number of total co-occurrences of words $x$ and $y$ in this order in a window size of 100 words.
} 


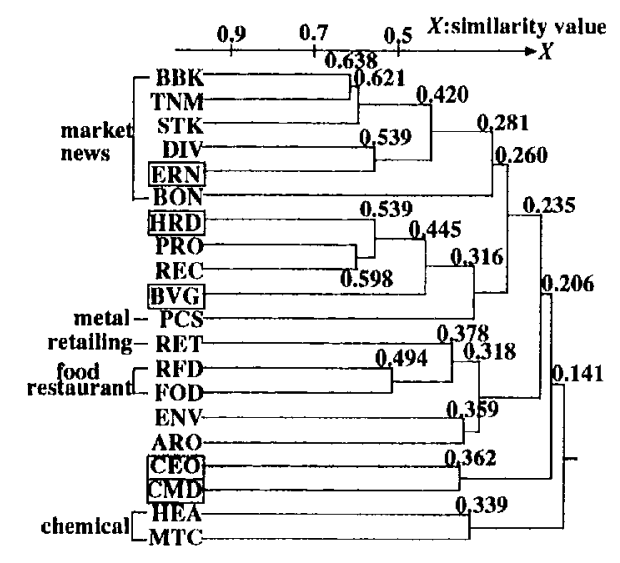

Figure 1: The results of 'Freq' experiment

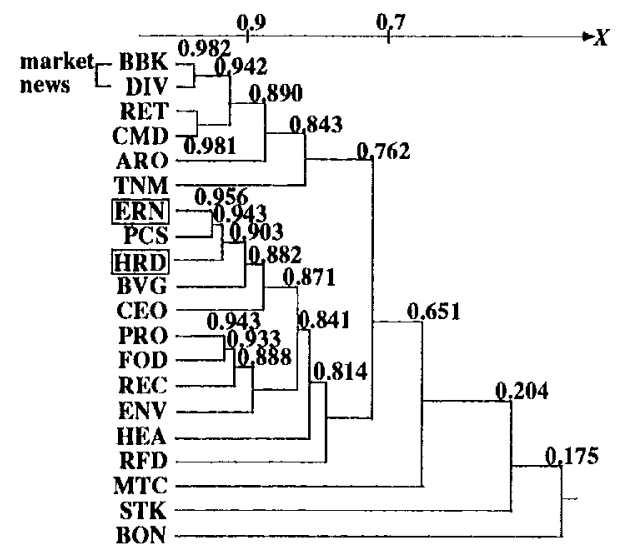

Figure 2: The results of 'Link' experiment

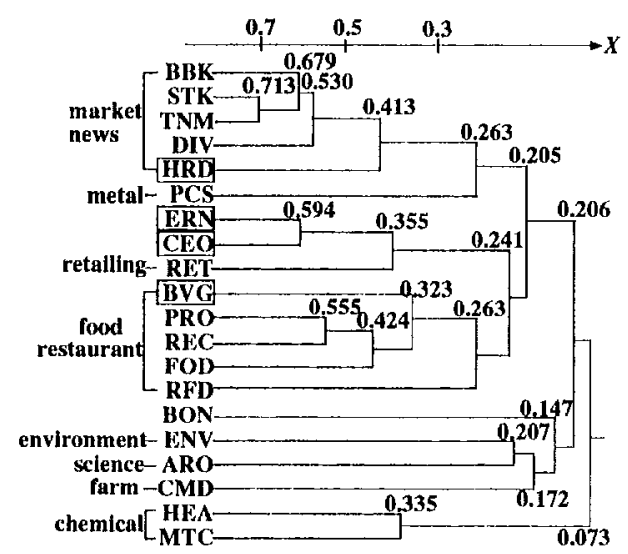

Figure 3: The results of 'Dis' experiment

\section{Discussion}

\section{WSD method}

According to Table 7 , there are 24 sets which could be clustered correctly in 'Dis', while 21 sets in 'Freq'. Examining the results shown in Figure 3, 'BVG' and 'HRD' are correctly classified into 'food - restaurant' and 'market news', respectively. However, the results of 'Freq' (Figure 1) shows that they are classified incorrectly. Table

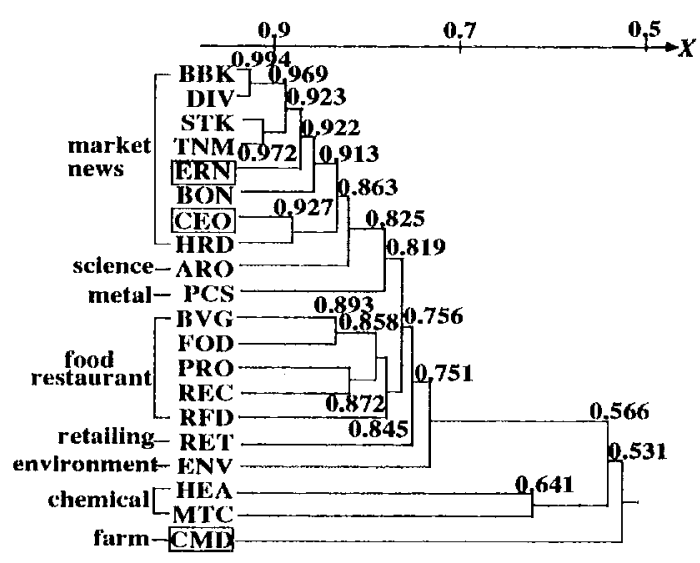

Figure 4: The results of 'Method' experiment

Table 8: Topic and category name

\begin{tabular}{l|l}
\hline Category & Topic \\
\hline market & BBK: Buybacks \\
news & BON: Bond Market News \\
& CEO: Dow Jones interview \\
& DIV: dividends \\
& ERN: carnings \\
& HRD: Heard on the street \\
& STK: stock market \\
& TNM: tender offers \\
\hline science & ARO: aerospace \\
\hline metal & PCS: precions metals, stones, gold \\
\hline food & BVG: beverages \\
restaurant & FOD: food products \\
& PRO: corporate profile \\
& REC: recreation, entertainment \\
& RFD: restaurant, supermarket \\
\hline retailing & RET: retailing \\
\hline environment & ENV: environment \\
\hline chemical & HEA: heall care providers, inedicine \\
\hline farm & MTC: medical and biotechnology \\
\hline
\end{tabular}

9 shows different senses of word in ' $B V G$ ', and 'HRD' which could be discriminated in 'Dis'.

In Table 9, for example, 'security' is high frequencies and used in 'being secure' sense in 'BVG' article, while 'security' is 'certificate of creditorship' sense in 'HRD'. One possible cause that the results of 'Freq' is worse than 'Dis' is that these polysemous words which are high-frequencies are not recognised polyseny in 'Freq'.

\section{Linking method}

As shown in Table 7, there are 23 sets which could be clustered correctly in 'Link', while 21 sets in 'Freq'. For example, 'ERN' and 'HRD' are both concerned with 'market news'. In Figure 2, they are clustered with high similarity value(0.943), while in Figure 1, they are not $(0.260)$.

Examining the results, there are 811 nouns in 'ERN' article, and 714 nouns in 'HRD', and 
Table 9: Different word-senses in BVG and HRD

\begin{tabular}{|c|c|c|}
\hline & $\overline{B V G}$ & HRD \\
\hline security & $\begin{array}{l}\text { the state of } \\
\text { being secure }\end{array}$ & $\begin{array}{l}\text { certificate of } \\
\text { creditorship) }\end{array}$ \\
\hline rate & a quantity in relation & price of charge \\
\hline $\begin{array}{l}\text { wale } \\
\text { stock }\end{array}$ & $\begin{array}{l}\text { the exchange of goods } \\
\text { total goods }\end{array}$ & $\begin{array}{l}\text { the anount of sold } \\
\text { stock nnarket }\end{array}$ \\
\hline
\end{tabular}

\section{Conclusion}

We have reported an experimental study for clustering of articles by using on-line dictionary definitions and showed how dictionary-refinition can use effectively to classify articles, each of which belongs to the restricted subject domain. In order to cope with the remaining problems mentioned in section 5 and apply this work to practical use, we will conduct further experiments.

of these, 'shares', 'stock', and 'share' which are semantically similar are inclucked. In linking method, there are 251 nouns in 'ERN' and 492 nouns in 'HRD' which are replaced for representative words. However, in 'Frec', each noun corresponds different coordinate, and regards to different meaning. As a result, these topies are clustered with low similarity value.

\section{Our method}

The results of 'Method' show that 31 out of 40 sets are classified correctly, and the pereentage attained was $77.5 \%$, while 'Freq', 'Link', and 'Dis' experiment attained $52.5 \%, 57.5 \%, 60.0 \%$, respectively. This shows the effectiveness of our method.

In Figure 4, the articles are judged to classify into eight catcgories. Examining 'FRN', 'CEO' and 'CMD' in Figure 1, 'CEO' and 'CMD' are grouped together, while they have different categories with cach other. On the other hand, in Figure 3, 'ERN' and 'CEO' are grouped together correctly. Examining the nomns which are belonging to 'ERN' and 'CEO', 'plant'(factory and food senses), 'oil'(petroleum and food), 'order'(command and demand), and 'interest' (delot and curiosity) which are high frequencics are correctly clisambiguated. Furthermore, in Figure 4, 'ERN' and 'CEO' are classified into 'market news', and 'CMD' are classified into 'far'm', correctly. For example, 'plant' which is used in 'factory' sense is linked with semantically similar words, 'manufacturing', 'factory', 'production', or 'job' etc.. In a similar way, 'plant' which is used in 'food' sense is linked with 'environment', 'forest'. As a result, the articles are classified correctly.

As shown in Table 7, there are 9 sets which could not be clustered correctly in our method. A possible improvement is that we use all definitions of words in the dictionary. Wo selected the first top 5 definitions in the dictionary for each nomn and used them in the experiment. However, there are some words of which the meanings are not included these selected definitions. This canses the fact that it is hard to get a higher percentage of correct clustering. Another interesting possibility is to use an alternative weighting policy, such as the widf (weighted inverse document frequency) (Tokunaga, 1994). The uidf is reported to have a marked actvantage over the idf (inverse document frequency) for the text categorisation task.

\section{References}

P. F. Brown ct al., 1991. Word-Sense Disambiguation Using Statistical Methods. In Proc. of the 29th Annual Meeting of the ACL, p1, 264-270.

F. Brill, 1992. A simple rule-based part of spech tagger. In Proc. of the 3 nd conference on applied natu. ral language processing, $\mathrm{ACL}, \mathrm{pp}$. 152-155. Trento, Italy, 1992.

K. W. Church et al., 1991. Using Statistics in Lexical Analysis, Lexical acquisition: Exploiting on-line resources to build a lexicon. (Zennik Uri (cd.)), pp. 115-164, Lonskon, Lawrence Erlbaum Associates.

L. Guthrie and E. Walker, "DOCUMENT CLASSI FICATION BY MACHINE: Theory and Practice", In Proc. of the 15th International Conference on Computational Linguistics, Kyoto, Japan, 1994, pp. 1059-1063

N. Jardine and R. Sibson, 1968. The construction of hicrarchic and non-hicarchic classifications. In Computer Journal, pp. 177-184.

K. S. Jones, 1973. A statistical interpretation of term specificity and its application in retrieval. Joumal of Documentation, 28 (1973) 1, 1). 11-21.

M. Liberman, editor. 1991. CD-ROM I, Association for Computational Linguistics Data Collection Initiative, University of Pennsylvania.

Y. Niwa and Y. Nitta, 1995. Statistical Word Sense Disambiguation Using Dictionary Definitions In Proc. of the Natural Language Processing Pacific Rim Symposium '95, Seoul, Korea, 1p. 665-670.

G. Salton and M. J. McGill, 1983. Introduction to Modern Information Retrieval. McGraw-Hill, 1983.

T. Tokunaga and M. Iwayana, 1994. T'ext Categorisation based on Weighted Inverse Document Frequency IPS.J SIG Reports, 94-NL-100, 1994.

D. Yarowsky, "Word sense disambiguation using statistical models of Roget's categories trained on large corpora", In Proc. of the 14th International Confer. ence on Computational Linguistics, Nantes, France, 1992, 1)1. 454-460

N. Yuasa et al, 1995. Classifying Articles Using Lexical Co-occurrence in Large Document Databases In Thans. of Information Processing Society Japan, p1). $1819-1827,36(1995) 8$.

D. Walker and R. Amsler, 1986. The Use of MachineReadable Dictionarios in Sublanguage analysis, $A n$ alyzing Language in Restricted domains, (Grishnam and Kittiedge (ed.)), pp. 69-84, Lawrence Erlbaum, Hillsclale, NJ. (1987) 2. 